Cipango
Cipango

$20 \mid 2013$

Nouveaux regards sur les arts de la scène japonais I

\title{
Le théâtre nō dans la Corée colonisée : du nō comme théâtre d'État
}

Nō Theatre in Colonial Korea: Nō as State Theatre

Johng Wan Suh

Traducteur : Pascal Griolet

(2) OpenEdition

Journals

Édition électronique

URL : https://journals.openedition.org/cipango/1923

DOI : 10.4000/cipango.1923

ISSN : 2260-7706

Éditeur

INALCO

Édition imprimée

Date de publication : 30 octobre 2013

ISSN : 1164-5857

Référence électronique

Johng Wan Suh, «Le théâtre nō dans la Corée colonisée : du nō comme théâtre d'État », Cipango [En ligne], 20 | 2013, mis en ligne le 16 avril 2015, consulté le 30 juin 2021. URL : http:// journals.openedition.org/cipango/1923 ; DOI : https://doi.org/10.4000/cipango.1923

Ce document a été généré automatiquement le 30 juin 2021.

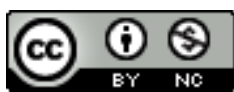

Cipango est mis à disposition selon les termes de la Licence Creative Commons Attribution - Pas d'Utilisation Commerciale 4.0 International. 


\title{
Le théâtre nō dans la Corée colonisée : du nō comme théâtre d'État
}

\author{
Nō Theatre in Colonial Korea: Nō as State Theatre
}

Johng Wan Suh

Traduction : Pascal Griolet

1 Les études sur le nō durant la période moderne sont très rares si on les compare à celles qui traitent de l'époque où vécut son fondateur Zeami 世阿弥 (1363-1443), ou même d'Edo (1603-1867)1. Les recherches sur sa diffusion dans les colonies japonaises sont quasi inexistantes. Non seulement on n'a pas encore pris en compte la dimension historique de cet art du spectacle, mais on ignore même ce qu'il fut concrètement alors. Ceci bien entendu s'applique également à la Corée ${ }^{2}$.

2 L'étude du nō dans les pays colonisés par le Japon revêt pourtant une grande importance, aussi bien pour l'histoire de cette tradition théatrale que pour celle de la colonisation. C'est dans cette double perspective que je me suis intéressé aux livrets de nō dont j'ai découvert l'existence à la bibliothèque de l'ancienne université impériale de Keijō [nom de Seoul durant la période coloniale]. C'est avec surprise en effet que j'ai constaté la présence ancienne sur la péninsule de ces livrets (vingt volumes, quatrevingt dix-sept numéros), ce qui suggère que le nō et les livrets de nō rencontrèrent un certain succès dans la Corée annexée par le Japon. Je me suis dans un premier temps limité à l'examen et aux données bibliographiques de ces cahiers, dans le cadre d'une recherche sur l'histoire du nō, sans encore faire de lien avec la colonisation de la Corée.

3 Le retard des recherches dans ce domaine est d'autant plus regrettable que les débats véhéments et les discussions sans fin sur la politique coloniale japonaise à l'égard de la Corée, et en particulier la politique d'assimilation et d'unification avec la métropole japonaise, ignorent très largement les réalités culturelles et la politique qui fut menée afin d'atteindre les objectifs de la colonisation. Non seulement pour le nō, mais pour toutes les autres formes d'arts scéniques, les études manquent cruellement sur la façon 
dont ils ont été introduits sur le sol des colonies, sur leur réception, sur les transformations qui ont pu se produire et enfin sur les publics qui furent visés. Or, il s'agit là d'un champ d'études qui devrait permettre d'examiner ce que fut concrètement l'état réel de la vie culturelle et de la politique appliquée en la matière.

4 Quelle fut l'importance du nō dans le contexte colonial ? Bien sûr, les arts du spectacle ont pour mission le divertissement du public et ne se mesurent pas uniquement à l'aune de critères politiques ou économiques. Il faut tout d'abord qu'il y ait une ouverture sur le plan culturel, une acceptation, une certaine sensibilisation. Il faut que soit établie une proximité, que soit créée une intimité. Mais dans le cas du nō, il y a un autre facteur important. Il s'agit de la proximité entre cet art et le pouvoir. Il faut en effet prendre en compte la protection qui lui fut accordée par les autorités politiques dès ses premiers temps, avec tout d'abord la passion du shōgun Ashikaga Yoshimitsu pour le tout jeune Zeami ${ }^{3}$, puis le goût du nō chez les maîtres du pouvoir, comme Oda Nobunaga et surtout Toyotomi Hideyoshi ${ }^{4}$, et enfin l'officialisation de cet art de la scène comme cérémonie rituelle de la classe des militaires, alors que se mettait en place le cloisonnement hermétique entre les quatre grandes classes de la société : militaires, agriculteurs, ouvriers et négociants.

5 L'histoire du nō, activité culturelle et divertissement de l'élite, révèle que celui-ci a toujours vécu à proximité du pouvoir. Sa transformation en art cérémoniel (shikigaku 式楽 $)^{5}$ chez les militaires lui fit perdre la liberté, la créativité et les innovations du temps de Zeami et lui conféra une nouvelle vie, différente, en tant que cérémonial. Une nouvelle force vitale, fondée sur la transmission et destinée à assurer sa longévité par la conservation, la répétition et la reproduction, remplaça le dynamisme de la force créatrice. Il faut garder ce point à l'esprit. Conservation et reproduction sont devenues les missions les plus importantes des familles ou des personnes héritières de cet art et on peut considérer qu'on est passé au cours de la période d'Edo de la dynamique d'une troupe d'acteurs à celle d'héritiers d'une tradition. De la même manière, la consignation par écrit d'une transmission orale peut être considérée comme un processus par lequel un art passe d'une vie créatrice à une autre fondée sur la transmission ${ }^{6}$. C'est à ce prix que le nō survécut dans la solennité durant l'époque d'Edo, mais son existence se trouva mise en danger, on le sait, par les violentes rafales des tempêtes que constituèrent les bouleversements et les profondes transformations de la société qu'inaugura la restauration de Meiji, en 1868.

6 De façon soudaine, le nō perdit les patrons qui l'avaient soutenu financièrement et avaient assuré son existence. Il lui fallut alors être reconnu comme forme artistique digne du Japon moderne. Il se redressa grâce à la classe la plus haute de la société, en particulier l'impératrice et certains membres de la noblesse impériale ${ }^{7}$, mais soulignons que ce salut ne répondait nullement à une demande venue de la population. Entre son effondrement et sa réhabilitation, on voit confirmée sous une forme renouvelée sa proximité avec le pouvoir. Or cette proximité se retrouve-t-elle de la même façon dans la Corée colonisée ? L'intimité entre le nō et ses spectateurs au Japon fut-elle de même nature dans la péninsule? Ces questions, sans doute naïves, ne peuvent-elles pas constituer un point de départ pour réfléchir à la signification du nō en Corée?

7 Comment fonctionna cette forme de théâtre propre au Japon, alors que celui-ci se constituait comme puissance en expansion colonisant les pays périphériques afin de devenir un empire colonial ? Put-il trouver sa place? Les Coréens pouvaient-ils y être 
réceptifs? Telles sont les questions que je me suis posées en menant mes recherches sur le nō dans la Corée colonisée.

\section{La splendeur d'un « théâtre d'État » (kokka geinō)}

8 La plus ancienne représentation de nō sur la péninsule coréenne remonte à 1616 (an 2 de l'ère Genna). Elle fut donnée dans le cadre de la cérémonie d'inauguration de la Maison japonaise (wakan/waegwan 倭館) de Pusan8.

Il faut ensuite mentionner une représentation de nō au Japon devant une délégation d'officiels coréens. Un document de 1643 (Kan.ei 30), les Notes journalières de l'ère Kan.ei (Kan.ei nikki 寛永日記), relate en détail les festivités organisées à leur intention. Le spectacle se déroula lors d'un banquet offert à la délégation qui comptait quatre cent soixante-deux émissaires et que conduisait l'ambassadeur coréen Yun Sun-ji 尹順之. Cette représentation, qui eut lieu le huit de la septième lune de cette année 1643, fut très soignée : y participèrent un maître de l'école Kanze, Kita Shichi 北七' ${ }^{9}$, et un maître de l'école Konparu 金春 ${ }^{10}$.

Pour l'époque moderne, les Notes journalières d'Umewaka Minoru (Umewaka Minoru jitsunikki 梅若実日記) constituent une source importante ${ }^{11}$. Elles attestent que les 20 et 21 mars 1904 se sont tenues chez maitre Kanze Kiyokado 観世清廉 (1867-1911) à Kyoto, en présence du chef de la délégation coréenne (kōshi/kongsa 公使) de la Corée, deux représentations de nō destinées à collecter de l'argent pour contribuer à l'effort de guerre contre la Russie. Celles-ci commencèrent à neuf heures du matin et se terminèrent à cinq heures du soir. Il s'agissait de réunir des fonds pour soutenir l'intendance de l'armée impériale. On récolta respectivement quatre cents et trois cents yens. Ce genre de représentation s'inscrit en fait dans la longue tradition des représentations destinées à réunir des fonds afin de venir en aide à un monastère ou un sanctuaire que l'on appelle Kanjin-nō勧進能, «nō de souscription ». Cette pratique était encore fréquente au début $\mathrm{du} \mathrm{xx}^{\mathrm{e}}$ siècle ${ }^{12}$.

11 Que signifiait donc la présence du chef de la délégation coréenne à cette séance de nō célébrée afin de récolter des fonds pour l'armée japonaise? Quelle fut sa propre contribution? En d'autres termes, combien a-t-il payé sa place? Comment fut initié alors ce type de séances dans le monde du nō ? Ces questions ne peuvent que susciter la curiosité et il faudra dans le futur tenter d'y répondre. Quoi qu'il en soit, il faut souligner ici que la relation entre le chef de la délégation et les acteurs de cette représentation ne fut pas simplement celle d'un spectateur face à des acteurs animés du seul objectif du plaisir d'une représentation.

12 De même, toujours selon les Notes journalières d'Umewaka Minoru, on trouve à la date du 13 avril 1905 la description qui suit :

Son Excellence monsieur Ǔiyang 義陽, Ambassadeur de Corée, a été invité au club Mitsui dans le quartier de Hibiya [à Tōkyō] pour un dîner et une séance de nō. On y a donné les pièces comiques Hashi-benkei 橋弁慶, Tsuchigumo 土蜘 et Kōyakuneri 膏 薬棟.

On peut lire encore à la date du 10 avril :

Kure Daigorō 吳大五郎 téléphona pour annoncer que Son Excellence monsieur Ǔiyang, Ambassadeur de Corée, et Son Éminence Yi Chae-gak 李栽覚, étaient invités à un dîner le 13 pour une séance de nō. 
Enfin, le 11 avril :

La séance de nō qui sera organisée chez Mitsui pour célébrer la guerre qui se déroule en Corée aura lieu devant Leurs Excellences Ǔiyan et Yi Chae-gak le $13 \mathrm{au}$ soir à $18 \mathrm{~h}$, avec au programme Hashi-benkei et Tsuchigumo.

On comprend ici que ces diplomates ont été invités à célébrer la victoire dans la guerre russo-japonaise. Ils ont sans doute été envoyés au Japon pour fêter la prise de PortArthur ou l'issue victorieuse de la bataille de Mukden. C'est donc dans un contexte hautement politique que «Son Excellence Monsieur l'Ambassadeur de Corée » a assisté en tant qu'« ambassadeur de la Corée dépêché pour fêter la victoire ». Ceci illustre bien la nature des relations entre les deux pays et le rôle que joua ici le nō.

Les premières séances de nō tenues devant des Coréens remontent donc à l'époque d'Edo, et on comprend que dans les temps anciens comme durant la période moderne les spectacles n'étaient pas donnés dans un lieu public ouvert à la population, mais à l'occasion de réceptions privées, et liées à un événement politique ou diplomatique. Ceci confirme la proximité du nō avec le pouvoir.

Près de quarante jours après cette séance organisée au Japon devant les représentants de la Corée afin de célébrer la victoire, le nō confirme définitivement son statut de "théâtre d'État ": une représentation est organisée dans la capitale coréenne, le 25 mai 1905, sur la place devant la gare de «la grande porte du Sud» (Namdaemun 南 大門), afin de célébrer l'inauguration de la ligne de chemin de fer entre la capitale et le port de Pusan. L'ouvrage intitulé le ?ō durant l'ère Meiji ${ }^{13}$ a déjà évoqué «la visite en Corée de Kanze Kiyokado » en s'appuyant sur des articles de quotidiens comme l'Osaka jiji shinpō 大阪時事新報, le Kyoto hinode shinbun 京都日出新聞 ou le Kokumin shinbun 国 民新聞. Je m'efforcerai donc de me référer le moins possible à ces derniers et privilégierai d'autres sources pour reconstituer le déroulement de cette cérémonie.

L'Histoire des chemins de fer coréens, éditée en 1929 par la Direction des chemins de fer du gouvernement général de Corée ${ }^{14}$, décrit en détail le déroulement de la cérémonie à laquelle assistèrent aussi les consuls des grands pays européens ainsi que des ÉtatsUnis. Il y est souligné la magnificence de l'événement, son caractère grandiose, « sans précédent dans l'histoire de Keijō ». Y sont aussi mentionnées la participation exceptionnelle de «Son Altesse » le prince Fushimi no miya Hiroyasuō 伏見宮博恭王 (1875-1946), qui représentait la famille impériale, et la présence de « Leurs Excellences coréennes Ǔiyang et Yi Chae-gak ». De nombreux discours furent prononcés, dont l'un du consul des États-Unis, puis se tint un banquet avec divers spectacles parmi lesquels nō, sumō, danses de type kagura ${ }^{15}$, tours de prestidigitation et ballets de danseuses coréennes. Tout ceci témoigne de l'importance que le Japon accorda à l'ouverture de cette ligne de chemin de fer. 


\section{1 : Cérémonie de nō}

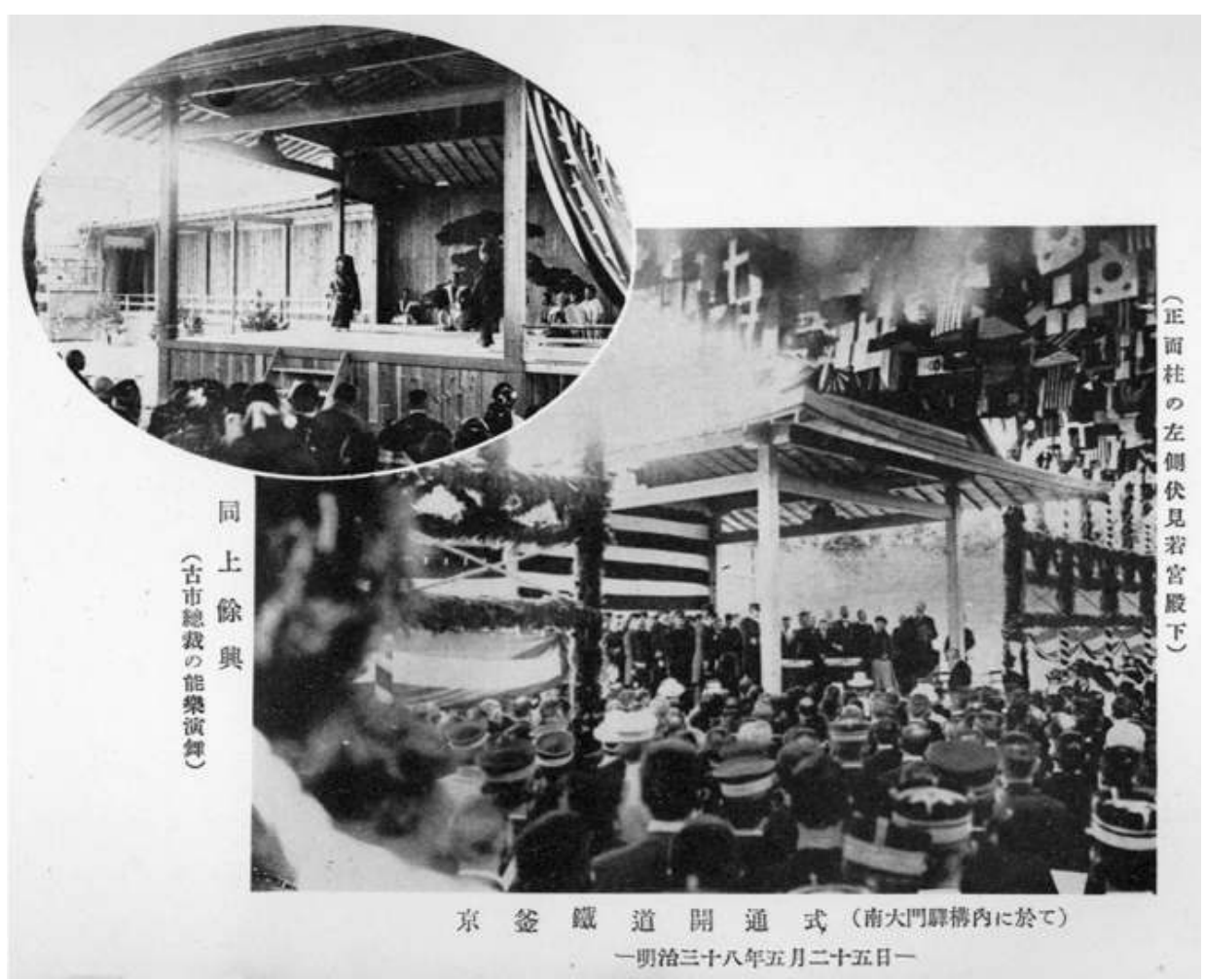

Cérémonie de nō à l'occasion de l'inauguration de la ligne de chemin de fer Séoul-Pusan. Séoul, Namdaemun, 25 mai 1905

Avec l'aimable autorisation de la bibliothèque de la Société d'études sur le génie civil (Doboku gakkai fûzoku doboku toshokan 土木学会付属土木図書館)

19 Les Nouvelles du chemin de fer (Tetsudō jihō 鉄道時報, $\mathrm{n}^{\circ}$ 296) du 20 mai 1905 présentent la liste des participants à cette cérémonie d'inauguration : vingt-neuf membres de la chambre des Nobles, cent quarante-cinq députés, dix-huit journalistes, six actionnaires, un chef d'entreprise et soixante-deux autres participants, soit en tout deux cent soixante-et-une personnalités. Manifestement, le Japon avait tout fait pour la réussite d'une cérémonie grandiose. Le document précise que certains participants venus du Japon ne purent assister à l'ensemble de la cérémonie : cinq membres de la chambre des Nobles, treize députés, deux banquiers, sept journalistes, un responsable des chemins de fer, soit vingt-huit personnes. Furent donc présents à la séance de nō trente-quatre membres de la chambre des Nobles et cent cinquante-huit députés, soit $24 \%$ de la chambre des Nobles et $53 \%$ de la chambre des Députés: il y eut manifestement une mobilisation générale du côté japonais.

Kinoshita Ikiru 木下生, journaliste des Nouvelles du chemin de fer, décrit dans son «Compte rendu sur la Corée » du 10 février 1905, la cérémonie comme un spectacle grandiose et conclut : « Le prestige de notre pays rayonne de plus en plus en Corée. »

La péninsule est présentée dans cet article comme un pays parfaitement souverain et indépendant grâce au Traité de Shimonoseki (avril 1895) qui a mis fin à la guerre sinojaponaise et a libéré le pays de la domination chinoise. Or, si la Corée avait bien retrouvé sa souveraineté face à la Chine, elle dépendait désormais du Japon et était bien loin de pouvoir constituer un État indépendant et souverain. Deux jours après cette cérémonie, le Japon allait envoyer par le fond la flotte russe de la Baltique et sortir 
victorieux de la guerre russo-japonaise. Le traité de Portsmouth (septembre 1905), puis le Traité de coopération et de commerce entre le Japon et la Corée (Kannichi kyōshō jōyaku 韓日協商条約, novembre 1905), connu aussi sous l'appellation du «Second traité de coopération nippo-coréen » (Dainiji nikkan jōyaku 第二次日韓条約), permettront au Japon d'instituer en Corée le système de résident général (Tōkanfu 統監府) et de prendre le contrôle de la diplomatie de l'« Empire de la grande Corée » (Daikan teikoku/ Taehan cheguk 大韓帝国). La voie était ainsi ouverte à l'annexion de la Corée qui aura lieu cinq ans plus tard. Si on réfléchit à ce processus historique, l'ouverture de la ligne de chemin de fer entre Keijō et Pusan permit bien «le rayonnement du prestige de notre nation en Corée». L'article de ce journaliste, de ce point de vue, est particulièrement révélateur.

C'est aussi la raison pour laquelle on ne peut négliger le fait que «l'événement le plus important de la journée » fut la représentation de nō donnée à cette occasion. En rivalité avec la Russie pour la domination de la Corée, l'armée japonaise se trouvait alors dans une situation très critique face aux forces russes. L'achèvement rapide de la ligne de chemin de fer reliant le port de Pusan à la capitale Keijō était d'une importance capitale pour assurer au Japon une supériorité stratégique. On nomma à la présidence de la compagnie des chemins de fer un certain Furuichi Kōi 古市公威, docteur en génie civil (kōgaku hakushi 工学博士). On verra plus loin le rôle que joua ce dernier dans l'organisation de nô lors de la cérémonie d'ouverture. Mais au-delà des considérations individuelles, quelle fut donc la signification de cette représentation? Bien entendu, le nō n'est pas un simple instrument politique. Mais on assiste ici à une étape importante de l'expansion du Japon impérial qui, après avoir gagné la guerre sino-japonaise, allait bientôt remporter la victoire sur la Russie. Le nō fut bien le «théâtre représentatif de l'État » qui permit de faire rayonner « le prestige de la nation » japonaise.

Après la restauration de Meiji, le nō avait failli disparaître. Grâce à la fondation en 1882 de la Société de nō (Nōgakusha 能楽社), il devint, à partir des années 1890, l'expression de la grandeur du Japon "État moderne », c'est-à-dire, de fait, du Japon impérial.

Ce passage d'un art de la scène rituel à un art de la scène étatique est en quelque sorte une réaffirmation, sous une forme moderne, de l'étroitesse des liens entre le nō et le pouvoir. Ce choix lui permit de survivre dans un nouveau contexte social et politique.

\section{Le nō de la cérémonie d'ouverture et le rôle joué par Furuichi Kōi}

La séance de nō qui s'est tenue lors de la cérémonie d'ouverture de la ligne de chemin de fer ne fut pas dirigée par maître Kanze Kiyokado, mais par le président du chemin de fer lui-même, Furuichi Kōi. Ce dernier était en effet très versé dans le nō, au point d'égaler les professionnels.

L'article cité plus haut du numéro 296 des Nouvelles du chemin de fer permet de connaître les détails du programme ainsi que le nom des acteurs prévus pour la séance du « nō rituel» (shikinō 式能 ${ }^{16}$ ) qui eut lieu le 25 mai et pour la séance du "nō caritatif» (jizen.nō 慈善能) du 26. La séance du 25 devait commencer à $13 \mathrm{~h}$ et enchaîner Yashima 屋島, interprété par Katayama Kurōsaburō 片山九郎三郎, Hagoromo 羽衣, par Kanze Kiyokado, et Kokaji 小鍛治, par Ōnishi Ryōtarō 大西亮太郎. Les intermèdes comiques étaient Kōyakuneri 膏薬棟, par Shigeyama Sengorō 茂山千五郎, et Chidori 千鳥, par 
Shigeyama Chūzaburō 茂山忠三郎. Avait lieu ensuite un dai-kagura 太神楽 ${ }^{17}$, suivi enfin de soixante-dix feux d'artifice lancés en plein jour et cent trente la nuit.

Le 26, à partir de dix heures du matin, étaient prévues les pièces qui suivent : Yamamba 山姥, par Kanze Kiyokado, Tamura 田村, par Ōe Matasaburō 大江又三郎, Mochizuki 望 月, par Ōnishi Ryōtarō, Tsuchigumo 土蜘, par Katayama Kurōsaburō, et Shōjō 猩猩, par Kanze Kiyokado. Les intermèdes comiques étaient Kazumō 蚊相缮, par Shigeyama Chūzaburō, Shūron 宗論 et Fusu 附子, par Shigeyama Sengorō. Suivait un banquet offert par la cour impériale coréenne dans le palais Kyŏngbok 景福宮, à trois heures de l'après-midi.

Le numéro 299 de la même revue, en date du 10 juin, fait toutefois apparaître des différences dans le programme et des changements dans la distribution des acteurs. Les trois pièces de nō du 26 auraient ainsi été suivies de "paroles propitiatoires » (shugen 祝言) et de deux intermèdes comiques: Dobukatsuchiri 井かつちり, par Shigeyama Chigorō, et Tachiubai 太刀奪, par Shigeyama Chūsaburō.

Qu'en fut-il en réalité ? Il est difficile de trancher et il faut procéder à des recoupements avec d'autres sources. Le premier article fut rédigé cinq jours avant les cérémonies, tandis que le second relate leur déroulement. Mais en fait, il semble que les représentations furent encore différentes. En effet, dans un ouvrage intitulé Furuichi Kōi édité en 1937 par l'« Association pour la mémoire de feu le Baron Furuichi Kōi » (Ko Furuichi danshaku kinen jigyōkai 故古市男爵記念事業会) et qui en retrace la vie, on peut lire ce qui suit :

Puis, le 25 mai 1905, il organisa à Keijō les cérémonies d'ouverture de la voie de chemin de fer. Il eut le grand honneur d'accueillir Son Altesse le prince Fushimi no miya Hiroyasuō et une délégation conduite par le ministre des Télécommunications Ōura Kanetake 大浦兼武 [1850-1918]. Les cérémonies furent suivies d'une grande réception. Le maître avait souhaité qu'à cette occasion se tienne une séance de nō et fit construire une scène devant la grande porte du Sud. Il fit venir de Tokyo, Kyoto et Osaka une vingtaine de maîtres de nō. Le jour de l'ouverture, à une heure de l'après-midi, la séance de nō commença par Yashima, pièce interprétée par Katayama Kurōsaburō de Kyoto, puis Hagoromo par Kanze Kiyotaka et enfin Kokaji par Ōnishi Ryōtarō. Le lendemain, il organisa une représentation de nō caritatif (jizen-nō) et interpréta lui-même Yamanba, qui fut suivie de Kumano par Kanze Kiyokado, puis de Tamura et Tsuchigumo par Katayama Kurōsaburō, et enfin Ran par Ōe Matasaburō. Toutes les pièces furent jouées de façon très raffinée et dans le style ancien, mais le jeu du maître dans la pièce Yamanba fut, dit-on, particulièrement éblouissant.

Si l'on se fie au numéro 296 des Nouvelles du chemin de fer, lors de la séance de nō caritatif du 26 Kanze Kiyokado joua Yamanba et Shōjō. Or il semble bien qu'en fait ce soit Furuichi qui ait interprété Yamanba. La raison de ce changement de programmation n'est pas claire. Toujours est-il que le quotidien Kyoto hinode shinbun du 4 juin 1905 indiquait :

Le deuxième jour, la séance commença à dix heures du matin et s'acheva à cinq heures de l'après-midi. La journée commença par Furuichi qui joua entre autres Yamanba. Les Japonais étaient nombreux et les Coréens rares, mais il semble que ces derniers aient bien apprécié les intermèdes comiques.

31 Le grand-père de Furuichi, qui occupait la fonction importante de comptable du fief de Himeji, organisait volontiers des séances de nō avec des personnalités importantes d'autres fiefs ou avec des négociants fortunés de la ville d'Edo. C'est ainsi que dès l'enfance Furuichi fut sensibilisé à cet art. Le 20 juin 1881, il devint disciple d'Umewaka 
Minoru (ill.2). Jusqu'à ce qu'il fasse partie de la délégation conduite par Yamagata Aritomo en Europe en 1888, et à l'exception de voyages à travers le Japon, il pratiqua le nō trois ou cinq fois par mois, parfois même sept ou neuf fois. Il interpréta lui-même une pièce de nō lors de sa propre cérémonie de mariage. Il avait installé une scène à son domicile et organisa souvent des séances de «nō mémoriels » (tsuizen-nō 追善能) pour commémorer des défunts. Enfin, il demanda à porter, à sa mort, le vêtement de cérémonie avec ses armoiries et un pantalon afin de pouvoir jouer du nō dans l'au-delà. Il aurait étudié ou pratiqué cent trente pièces du répertoire.

\section{2 : Umewaka Minoru.}

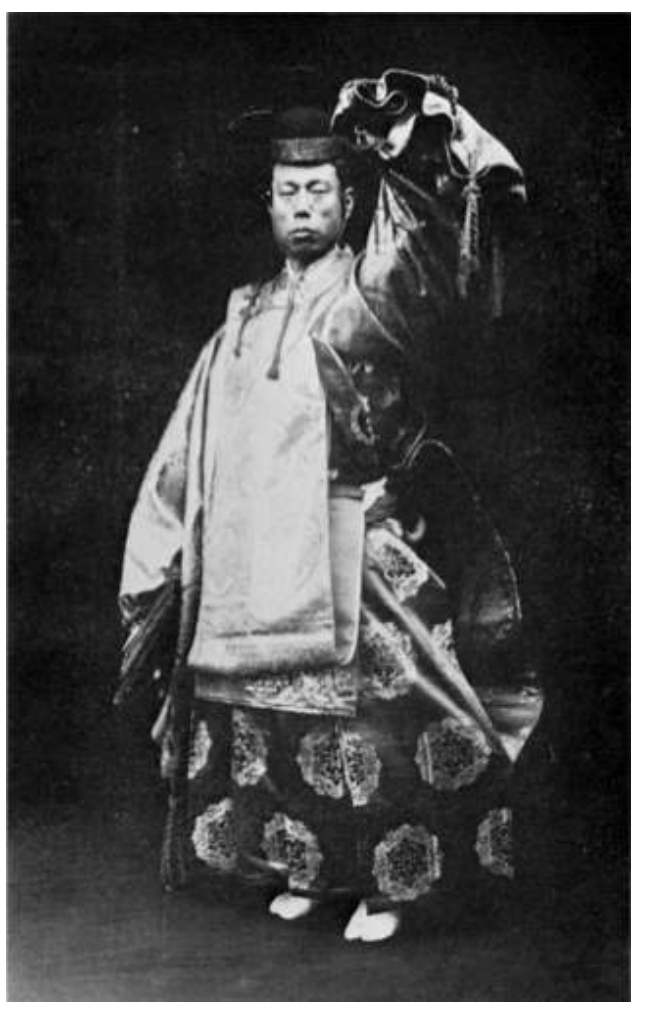

Avec l'aimable autorisation de la bibliothèque de la Société d'études sur le génie civil (Doboku gakkai fûzoku doboku toshokan 土木学会付属土木図書館)

Furuichi était donc un passionné de nō et son niveau artistique était équivalent à celui d'un professionnel. Ce que l'on doit remarquer ici, c'est qu'au deuxième jour de la cérémonie d'inauguration, lors du nō caritatif, Furuichi interpréta lui-même Yamanba, pièce à laquelle il était très attaché (ill. 3). Le quotidien Osaka jiji shinpō daté du 2 mai 1905 rapporte :

Kanze Kiyokado, qui a attiré sur lui l'attention du monde du théâtre nō du Kansai pour son interprétation d'un nō rituel lors des funérailles de Kanze [Kiyotaka, son père], réside actuellement à Kyoto, mais il est invité par Monsieur Furuichi Kōi à se rendre en Corée le 25 et le 26 de ce mois pour l'inauguration de la ligne de chemin de fer entre Keijō et Pusan. Furuichi est certes un amateur, mais il maîtrise parfaitement les arcanes du nō. C'est un homme ambitieux et imbu de lui-même. Il veut être admiré des connaisseurs du nō chez les ressortissants japonais résidant en Corée, en présentant des pièces « au charme subtil » [yūgen] comme Bashō 芭蕉 ou Yugyō yanagi 遊行柳. La pièce qui lui est la plus chère est Yamanba. On peut se demander si Kanze Kiyokado, qui est considéré comme le Kawakami Otojirō du théâtre $n \bar{o}^{18}$, connaît les motifs secrets de M. Furuichi qui doit être fou de joie à 
l'idée que c'est à lui que reviendra la gloire d'avoir accompli le vœu nourri autrefois par Toyotomi Hideyoshi, mais jamais réalisé jusqu'à sa mort, de se rendre en Corée avec les plus grands maîtres de l'école Kitaryū 喜多流. Toujours est-il qu'héritier de la prestigieuse famille Kanze, Kiyokado voit dans cette représentation en Corée une occasion d'attirer l'attention et de faire rayonner la gloire de son école. Considérant qu'une telle occasion de se produire en Corée ne se présenterait peut-être pas deux fois, il se prépare à partir en invitant à l'accompagner ceux qui voudraient le suivre...

\section{3 : Furuichi Kōi}

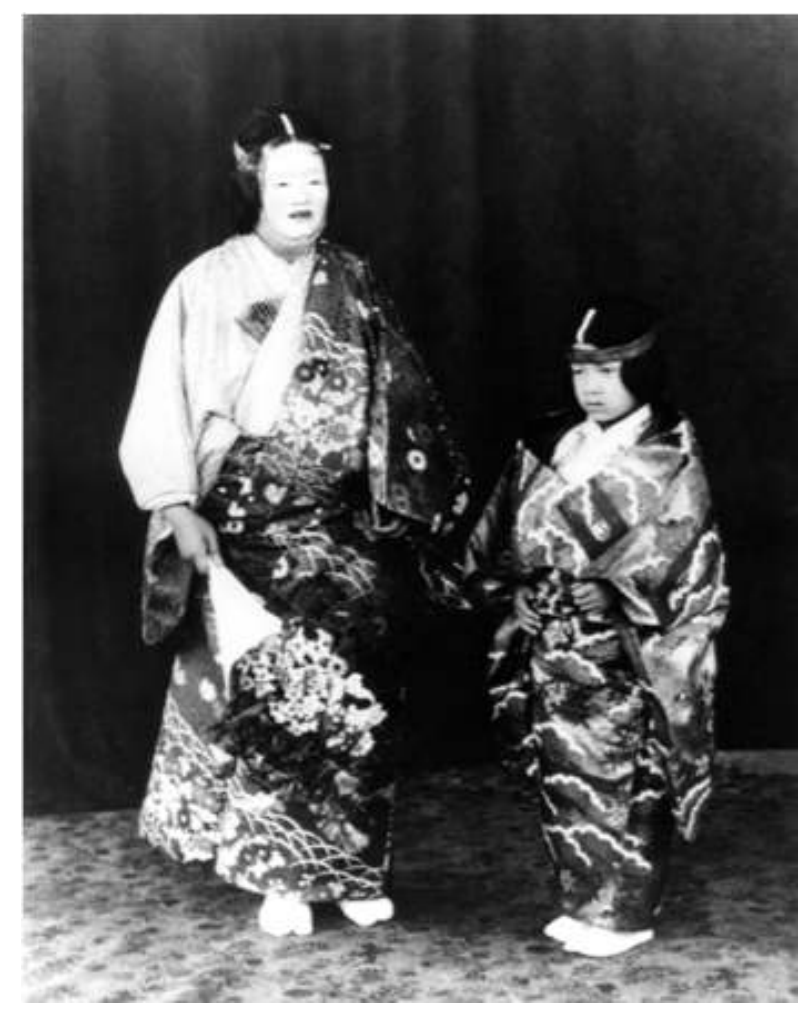

Furuichi Kōi, interprète de la pièce Yamanba

Avec l'aimable autorisation de la bibliothèque de la Société d'études sur le génie civil (Doboku gakkai fûzoku doboku toshokan 土木学会付属土木図書館)

On voit ici à quel point la pièce Yamanba était chère à Furuichi. Sa biographie la mentionne parmi «les pièces que jouait le mieux monsieur Furuichi $»^{19}$. Mais le plus remarquable, c'est que Furuichi a organisé la représentation en tant que président et que c'est lui, le président en personne, qui se montra sur scène jouant sa pièce préférée. Est-ce qu'un autre président aurait pu avoir l'idée d'organiser une représentation de nō pour la cérémonie d'inauguration de cette ligne de chemin de fer? Et dans ce cas, la représentation aurait-elle été si grandiose?

Pour tenter de répondre à ces questions, considérons de nouveau un passage des Notes journalières d'Umewaka Minoru, dont Furuichi avait été le disciple, à la date du 19 mai 1905 :

Kiyokado est parti en train hier soir depuis la gare de Shinbashi. Il se rend à Moji pour aller en Corée où il participera à deux journées de nō. Il répond ainsi à une demande de Furuichi Kōi. Celui-ci organise une séance de nō pour la cérémonie d'inauguration de la ligne de chemin de fer entre Keijō et Pusan. Bien que surpris par cette demande inattendue, Kiyokado a fait le nécessaire pour répondre à la 
demande de Furuichi et s'est entouré de vingt-quatre personnes pour mener à bien cette représentation (...). japonais, Kōkuri 高句麗, Shiragi 新羅 et Kudara 百済. Que savait donc Kiyokado de ces «trois royaumes", de la Corée et de son histoire? On considérait alors comme des vérités historiques les passages des Annales du Japon (Nihon shoki) qui présentent la Corée comme un pays soumis au Japon à l'époque antique. Or les Annales se fondaient sur un ouvrage intitulé La conquête des trois royaumes coréens (Sankan seibatsu 三韓征伐) qui s'est révélé être un faux. C'est en s'appuyant sur ce faux que furent justifiées les invasions japonaises de 1592 et de 1596, connues en Corée comme les "Troubles causés par les Wa l'année du dragon de l'eau » (Jinshin waran/Imjin waeran 壬辰倭乱). C'est de nouveau ce texte et le terme de «trois royaumes » qui furent utilisés au début de l'ère Meiji pour justifier les projets de conquête de la Corée, puis l'annexion de l'Empire de la grande Corée, puis encore l'émergence de la théorie d'une même origine ethnique des Japonais et des Coréens, et enfin la politique d'assimilation des Coréens à l'empire japonais.

37 Par ailleurs, observons que dans la première partie de cet article de l'Osaka jiji shinpō, lorsqu'il est question de Furuichi et de son art, on est dans l'ordre du " privé », tandis qu'ensuite il semble que l'on entre dans l'ordre du " public », avec la vision de l'histoire d'un État-nation et l'expression d'un "patriotisme » officiel. Au début, le discours fait référence uniquement aux Japonais: les «connaisseurs du nō chez les ressortissants japonais résidant en Corée ». Ensuite, il renvoie à un peuple autre, à une culture autre, à un pays autre. Le ton n'est plus le même. Si l'on considère qu'on est à la veille de l'annexion de la Corée, on pourrait considérer qu'il est fait référence au projet « d'invasion ». L'article de l'Osaka jiji shinpō suggère que c'est là le point de vue de Kanze Kiyokado. Il faut ici constater que la presse et les journalistes se faisaient ainsi les porte-parole de l'idéologie de «la conquête des trois royaumes coréens » enracinée dans une vision impérialiste japonaise. Toujours est-il qu'il faut souligner le décalage entre la première et la seconde partie de cet article.

Les pièces de nō furent jouées lors de la cérémonie d'ouverture de la ligne de chemin de fer sur une scène certes provisoire, mais une scène de nō authentique. Dans le numéro 299 des Nouvelles du chemin de fer, l'article intitulé "Coup d'œil sur la Corée $\mathrm{n}^{\circ}$ 6, Visite de Keijō » raconte ainsi :

Avant-hier, j'ai passé une nuit à l'auberge Mikazuki. Dès le lendemain matin, j'ai quitté d'auberge pour être hébergé par le groupe Kajima. Je me suis tout d'abord rendu sur le site où se préparait la cérémonie d'ouverture de la ligne de chemin de fer, qui se déroulera en plein air devant la grande porte du Sud. J'ai rencontré le président M. Furuichi, le directeur M. Kawasaki, le chef de la commission des préparatifs de la cérémonie d'ouverture, M. Kasai. Ils sont tous extrêmement occupés. J'ai jeté un coup d'œil sur le lieu de la cérémonie et j'ai été surpris de son 
envergure. Le portail d'entrée est grandiose, les tables pour les repas sont disposées dans un espace très spacieux, une authentique scène de nō a été construite. Ces préparatifs augurent que les Coréens seront émerveillés.

39 On peut voir dans la dernière phrase l'expression de la fierté ressentie devant la grandeur du lieu et la scène de nō tout en jetant sur les Coréens un regard empreint d'une sorte "d'orientalisme ${ }^{20}$. Cette fierté ressentie devant la scène du "théâtre représentatif de l'État » est si grande qu'elle reparaît à plusieurs reprises dans la suite de l'article ${ }^{21}$ :

Leurs deux Excellences sont, de façon exceptionnelle, venues voir l'état des préparatifs de la cérémonie. Elles ont pensé que cela devrait réjouir les gentilshommes et les nobles dames résidant en Corée. Ensuite, une scène de nō authentique a été construite et a été conçue pour la participation de Leurs Excellences. (...)

Les bâtiments, et en particulier le grand portail d'inauguration à l'entrée du lieu, sont si grandioses qu'on ne pourrait guère leur trouver d'équivalent à Tōkyō. Les tables pour les repas sont disposées dans un grand espace qui est magnifiquement décoré. Le lieu même de la cérémonie n'est pas très grand mais la perfection de la construction de la scène du nō a dû demander beaucoup de travail. (...)

Des Coréens curieux et insouciants sont venus en foule, les uns derrière les autres, voir la cérémonie d'ouverture de ce jour et cette foule a recouvert la dizaine d'hectares de la place. Vus de haut, ce sont des têtes noires vêtues de blanc. On a l'impression d'être devant un rassemblement de grues et de hérons. C'est sans doute la première fois qu'un tel rassemblement a lieu dans l'histoire de Keijō.

Ces passages sont également élogieux pour la scène "authentique » de nō. Le regard jeté sur les Coréens est empreint de cette condescendance qui caractérise le regard orientaliste. Il ressort de ces différents articles que cette représentation du nō à Seoul fut perçue comme une expansion du nō en Corée et qu'elle s'inscrivait dans la logique de conquête de la péninsule. Elle est présentée comme un exploit qu'aurait voulu réaliser Toyotomi Hideyoshi. On peut y lire un témoignage de cette vision de l'État japonais et de la Corée qui se constituaient alors comme idéologie. Dans ce contexte, l'installation d'une scène de nō authentique était le symbole du prestige du Japon impérial.

41 À son retour au Japon, l'un des interprètes de kyōgen, Shigeyama Sengorō, évoque cette scène "authentique »: "La scène de Keijō a été construite tout en cyprès du Japon (hinoki), sauf les piliers du pont, faits en bambou. » Le caractère authentique de cette scène doit-il être réduit à l'exaltation d'un orientalisme, d'une idéologie, d'une conception de l'État? Le nō en 1905 avait déjà acquis le statut de «théâtre d'État ». Il avait donc été reconnu comme spectacle pouvant accompagner la cérémonie d'ouverture de la ligne de chemin de fer. Autrement dit, il était reconnu comme une forme artistique digne d'être présentée aussi bien à l'intérieur qu'à l'extérieur du pays pour représenter le Japon, État moderne. Parallèlement, il ne faut pas minimiser le rôle joué par Furuichi Kōi, passionné du nō, dans le choix de pièces de nô lors de la cérémonie d'ouverture. L'intérêt et l'admiration qu'il manifestait à cet art n'étaient pas ordinaires. C'était bien pour lui « le plaisir d'un gentilhomme». Si l'on se fie à l'article de l'Osaka jiji shinpō, on peut considérer qu'il avait l'intention de se présenter sur scène dès les premières étapes du projet de cette représentation de nō. 


\section{Conclusion}

Sept mois après l'inauguration de la ligne de chemin de fer entre Keijō et Pusan, le décret impérial numéro 243 du 21 décembre $1905^{22}$ institua le régime du résident général de Corée et déposséda la Corée de son indépendance sur le plan diplomatique. Un an plus tard, le 30 mars 1906, fut promulguée une loi permettant l'achat de la ligne de chemin de fer, dont la gestion fut transférée au gouvernement général de Corée le $1^{\mathrm{er}}$ juillet de la même année. Enfin, 4 ans plus tard, le $1^{\mathrm{er}}$ octobre 1910, la Corée fut annexée, perdit toute indépendance et devint colonie japonaise.

Considérant le contexte historique dans lequel cette cérémonie s'inscrivit, force est de constater qu'elle revêtit une grande importance aussi bien sur le plan symbolique que politique et diplomatique, sans parler de la signification stratégique de l'ouverture de la ligne de chemin de fer qu'elle célébrait. La cérémonie fait date dans l'histoire du nō en ce qu'elle rassembla un quart des membres de la chambre des Nobles et plus de la moitié des députés de la Chambre basse. La représentation de nō, dont on a dit qu'elle fut considérée comme « la plus grande attraction » de cette cérémonie très importante, atteste que le nō avait déjà le statut particulier d'art de la scène représentant l'État japonais et qu'il pouvait témoigner de la grandeur du Japon impérial.

Pour cette représentation fut érigée une scène de nō " authentique ", sans doute la plus authentique de toutes celles qui furent édifiées par la suite en Corée. Mais il semble que celle-ci ait été démontée après les cérémonies. En effet, un article du Keijō nippō 京城日 報 (Les nouvelles de Keijō) daté du 10 janvier 1916 fait allusion à la vogue du chant de nō à Keijō et laisse entendre qu'il n'existait pas encore de scène de nō utilisable dans la capitale :

Avec ces progrès et la vogue du nō et du kyōgen, on peut penser que les amateurs envisageront dans un futur proche l'édification d'une scène de nō.

L'installation d'une scène authentique pour ce spectacle avait non seulement pour but de marquer l'importance de la cérémonie, mais résultait aussi du désir de Furuichi, amateur passionné du nō, de monter lui-même sur scène et de présenter sa pièce favorite, Yamanba à cette occasion. Il faut donc ici aussi voir la passion d'un individu et son souci de la perfection.

Que signifia donc cette représentation de nō pour les Coréens? Comment fut-elle perçue? Mis à part les officiels coréens, la plupart des participants étaient japonais. Nous avons vu que l'article du quotidien Kyoto hinode shinbun qui décrivait la foule qui grouillait dans la ville de Keijō précisait que «les [spectateurs] Japonais étaient nombreux et les Coréens rares. » Les Coréens, qui ne comprenaient pas le japonais et ignoraient l'histoire et la culture du Japon, ne pouvaient bien sûr pas comprendre des pièces comme Kokaji, où un forgeron aveugle reçoit l'ordre de l'empereur Ichijō 一条 de lui forger un sabre, ou Mochizuki, qui évoque la vengeance des frères Soga. Ils ne pouvaient pas non plus apprécier Tamura ou Yashima, pièces qui évoquent des figures historiques liées à l'épopée du clan des Taira.

Si les Coréens "[ont] bien apprécié les intermèdes comiques", cela signifie qu'ils réagirent en riant à la gestuelle cocasse de Kazumo, ou devant le comportement des serviteurs qui mangent en cachette du sucre dans Busu. Autrement dit, pour le public coréen de l'époque, le nō n'était pas un spectacle de divertissement. Mais en fait, il en allait de même au Japon. 


\section{dans des salles de théâtre ».}

L'opéra de Russie a rencontré un succès tout à fait inattendu [au Japon]. Le Théâtre impérial (Teikoku gekijo 帝国劇場) compte donc inviter, en février ou en mars prochain, une troupe italienne d'opéra et envisage pour l'avenir de convier les grands opéras du monde entier afin de populariser cet art lyrique. Nombre d'amateurs de théâtre souhaitent que tout en présentant au public japonais les opéras du monde entier, on présente aussi des pièces de nō, qui est l'opéra propre au Japon ${ }^{23}$. À ce sujet, le directeur du théâtre, M. Yamamoto, déclare :

«Je souhaiterais que le nō soit joué dans cette salle et j'ai d'ailleurs approché certains maîtres trois ou quatre fois grâce au truchement de certaines de mes connaissances pour connaitre leurs dispositions, mais ils ont leur fierté et n'envisagent pas de s'abaisser à jouer dans une salle de théâtre. Je pense que nous vivons dans une époque très différente des temps anciens et qu'il serait très profitable de plusieurs points de vue de présenter le nō au grand public et ne pas le confiner au divertissement exclusif des riches. Le Théâtre impérial est en tout cas toujours prêt à réserver le meilleur accueil à ces grands maitres s'ils sont disposés à se présenter ici.»

Pourtant Umewaka Rokurō répondit de façon catégorique : «Je refuse absolument cette proposition, ce n'est pas mon vœu. » Il ne s'expliqua pas sur les raisons de ce refus mais il nourrissait sans doute au fond de lui le préjugé ancien qui considère les acteurs de théâtre comme des gueux ou des «mendiants des bords de rivière " (kawara kojiki 河原乞食). Il ne souhaitait donc pas populariser l'art du nō.

Un célèbre amateur de théatre s'en offusqua et déclara, furieux :

«Durant l'époque Edo, les interprètes de nō protégés par le gouvernement traitaient les acteurs de théatre de "mendiants des bords de l'eau". Mais comme le dit le proverbe "les interprètes du nō sont comme des singes", pour la plupart d'entre eux ils vivent toujours aux crochets des aristocrates et des riches et ont du mal à se libérer de cet atavisme. Un art qui dépend en totalité de protecteurs fortunés ne peut être considéré comme un art véritable. Aujourd'hui même, les riches qui semblent protéger les interprètes du nō ne cherchent en réalité qu'à faire figure de généreux mécènes. C'est une honte pour un artiste de se complaire dans une telle situation et pour l'avenir du nō aussi il faut absolument que les artistes ne soient plus dépendants de riches protecteurs et s'adressent à un plus large public. Les interprètes du nō se cachent afin de se protéger, car ils ont très peur que si le nō se popularise, le protocole scénique et l'interprétation ne soient déformés, mais là encore ils ont tort. C'est en se popularisant que le nō pourra progresser et devenir un véritable opéra. »

C'est ainsi que les défenseurs d'un renouveau crient que le temps est arrivé pour que les interprètes du nō ne restent plus dans leur cocon mais procède enfin à leur mue.

Outre la question de la réforme du nō et de sa représentation au Théâtre impérial, cet article du Tokyo nichinichi shinbun montre bien que le nō, qui avait perdu sa raison d'être après la restauration de Meiji, survécut en tant que " théâtre d'État » et éprouva dès lors les douleurs de l'accouchement que suscitait une demande pressante venue de l'extérieur pour qu'il devienne un « théâtre du peuple » ou « de la nation »" 


\section{NOTES}

1. Cet article a été publié en japonais sous le titre «Shokuminchi chōsen ni okeru nō - Keipu tetsudō kaitsū shikiten ni okeru 'kokka geinō’ nō » 植民地朝鮮における能一京釜鉄道開通式典 における「国家芸能」能 (le Nō dans la Corée colonisée - La séance de nō, art de la scène de l'État, à l'occasion de la cérémonie d'ouverture de la ligne de chemin de fer Séoul-Pusan ", dans un numéro spécial de Ajia Yūgaku アジア遊学 intitulé Shokuminchi chōsen to teikoku Nippon, minzoku toshi bunka 植民地朝鮮と帝国日本一民族 - 都市 - 文化 (la Corée colonisée et le Japon impérial population, villes, cultures), Bensei shuppan 勉誠出版, $\mathrm{n}^{\circ} 138,2010$, p. 134-153. L'auteur est professeur au département d'Études japonaises de l'université Hallym 翰林 (Corée), directeur du centre de recherche sur le Japon de cette même université.

2. À ma connaissance, il n'existe sur cette question que les trois articles que j'ai publiés (en coréen) sous le titre « Recherche fondamentale sur les livrets de nō (utaibon 謡本) conservés à la bibliothèque de l'université de Séoul » (jap. : Souru daigaku zō utaibon no kisoteki kenkyū ソウル大 学蔵謡本の基礎的研究), in Nagoya geinō bunka-kai (éd.), Nagoya geinō bunka 名古屋芸能文化, $n^{\circ}$ 6, 1997; $n^{\circ}$ 9, $1999 ; n^{\circ}$ 13, 2003), auxquels il faut adjoindre «Aspects de la réception du nō en Corée - Annuaire des praticiens du nō résidant en Corée » d'O Hyŏn-yŏl 蛬鉉烈 (Kankoku tōchi nōgaku kyōju no shosō, kō-zaikankoku yōkyokuka meikan 韓国当地能楽享受の諸相、稿 - 在韓国謡 曲家名鑑, en coréen, in Hōseidaigaku daigakuin kiyō 法政大学大学院紀要, $n^{\circ} 43,1999$ ) et «les Représentations du nō en Corée durant les dernières années de l'ère Meiji - Autour de la représentation organisée par la revue Kokufū 国諷 en 1910 ” d'Iizuka Erito 飯塚恵理人 (Meiji matsunen no kankoku ni okeru nōgaku kōen - Meiji yonjūsannen «kokufu » nōgaku kōen o chūshin ni - 明 治末年の韓国における能楽公演 一 明治四十三年「国諷」能楽講演を中心に, in Kanrin daigakkō nihongaku kenkyūsho 翰林大学校日本学研究所, Kanrin nihongaku 翰林日本学, $n^{\circ} 14,2008$, en japonais). Le premier s'appuie sur une recension d'articles relatifs à la Corée publiés au Japon entre 1915 et 1945 dans des revues spécialisées comme Nōgaku 能楽 (le Nō), Nōgaku jihō 能楽時報 (la Revue du nō) et Nōgaku shinpō 能楽新報 (les Nouvelles du nō). Ces données, qui mériteraient toutefois d'être complétées, sont très utiles pour avoir un premier aperçu du développement du nō en Corée. Il ne s'agit néanmoins que d'une compilation de documents. Le second tient mal compte de la situation dans laquelle se trouvait la Corée à la fin de l'ère Meiji et ne mesure pas la dimension historique de la représentation de nō. Il est donc insatisfaisant dans la mesure où l'étude du nō dans les colonies doit s'inscrire dans l'étude de la colonisation japonaise et ne pas s'arrêter simplement à l'analyse d'une forme théâtrale.

3. NDT : on sait que c'est en 1374 (Ōan 7) que le chef d'une troupe d'acteurs itinérants connu plus tard sous le nom de Kan.ami 観阿弥 (1333-1384), accompagné de son fils de douze ans, le futur Zeami, donna une représentation de sarugaku 猿楽, divertissements qualifiés littéralement de «singeries ", devant le troisième shōgun de la dynastie des Ashikaga, Yoshimitsu (1358-1408), alors âgé de seize ans. La représentation eut lieu au sanctuaire d'Imagumano 新熊野, dans la capitale impériale. Yoshimitsu fut vivement impressionné par la beauté de l'enfant exécutant avec son père la danse rituelle d'okina « le vieillard » et décida de prendre cette troupe sous sa protection. Quatre ans plus tard, en 1378, Yoshimitsu assistait à la fête de Gion, Zeami assis à côté de lui. Cela suscita des critiques de la part des nobles, choqués de ces égards pour un "acteur ", c'est-à-dire un « gueux ». Mais Zéami fut couvert de présents par le shōgun, et les vassaux de ce dernier se virent eux aussi contraints de le traiter avec les plus grands égards, puis de protéger le nō.

4. NDT : Toyotomi Hideyoshi (1537-1598) se passionna pour ce genre théâtral au point de s'y exercer et d'interpréter lui-même des pièces dans la dernière partie de sa vie. Il se conduisit aussi en mécène attentionné des quatre troupes dites des «singeries de la région du Yamato » Yamato 
sarugaku (Kanze 観世, Hōshō 宝生, Konparu 金春 et Kongō 金剛). Cette politique de soutien au nō sera poursuivie par les Tokugawa et les seigneurs locaux durant toute l'époque d'Edo.

5. NDT : le terme désigne un nō, danses et chant, donné lors d'une cérémonie publique liée à l'exercice du pouvoir politique. Le nō prit plus particulièrement ce rôle à l'époque d'Edo.

6. NDT : voir à ce sujet René SIEFFERT, la Tradition secrète du nō , suivi de Une journée de nō, Gallimard/Unesco, 1960, ainsi que Sakae MURAKAMI-GIROUX, Zeami et ses Entretiens sur le nō, Presses orientalistes de France, 1991.

7. NDT : Iwakura Tomomi 岩倉具視 (1825-1883), noble qui joua un rôle politique de premier plan lors de la restauration de Meiji, organisa chez lui en 1876 une séance de nō en présence de l'empereur et, en 1879, une autre séance devant le général Grant (1822-1885), futur président des États-Unis. Il aurait eu à chaque fois pour objectif de rehausser le prestige de cet art de la scène qu'il pratiquait lui-même. Son cousin, Bōjō Toshitada 坊城俊政 (1826-1881), chef du protocole au palais impérial, le seconda dans ses efforts pour sauver cet art de la scène traditionnel.

8. NDT : sur les échanges entre Japon et Corée à l'époque d'Edo, voir le dossier « la Péninsule retrouvée ", Cipango n ${ }^{\circ} 17,2010$. Le rôle du port de Pusan et des comptoirs japonais est évoqué en particulier dans l'article de Guillaume CARRÉ.

9. Il s'agit d'un maître de l'école connue sous le nom de Kitaryū 喜多流.

10. La représentation commença par les danses propitiatoires que l'on appelle shikisanba 式三番 et sanbasō 三番舅, exécutées par Den.emon 层右衛門. Elles furent suivies par la célèbre pièce Takasago 高砂 interprétée par le maître Kanze après qu'il en eut donné des explications. L'intermède comique (kyōgen) fut la pièce Ebisu Bishamon 夷昆沙門. Le maître Shichi 七 présenta de son côté la pièce Momijigari 紅葉狩, qui fut suivie d'un nouvel intermède comique, Utsubozaru 䩭猿. Enfin le maître de l'école Konparu présenta la pièce intitulée Yōrō 養老. Certaines de ces pièces, comme Takasago ou Utsubozaru, ont été traduites en français par René SIEFFERT, Nō et kyōgen, op. cit..

11. Umewaka Minoru (1828-1909), acteur de nō de l'école Kanze, concourut à surmonter la crise que traversa ce théâtre lors de la modernisation du pays notamment en construisant des salles de nō et en organisant des représentations publiques payantes.

12. Les notes attestent ainsi que le 26 du même mois, eut lieu une représentation de nō poursuivant le même objectif à la « capitale de l'ouest », c'est-à-dire à Kyoto ( "Chez les Katayama de Kyoto s'est tenue une représentation destinée à financer l'intendance militaire »), et pour le lendemain, le 27 mars («Une séance de nō destinée à aider financièrement l'armée pour la guerre russo-japonaise eut lieu chez moi »).

13. KURATA Yoshihiro 倉田喜弘 (dir.), Meiji no nōgaku 明治の能楽, Nihon gakujutsu shinkōkai 日 本学術振興会, 1994-1997, et particulièrement le vol. III, 1996.

14. Chōsen sōtokufu tetsudō kyoku kankō 朝鮮総督府鉄道局刊行, Chōsen tetsudō shi 朝鮮鉄道史, Keijō,1929.

15. NDT : danses propitiatoires.

16. NDT : ce terme désigne les nō qui étaient joués sur une scène du palais shōgunal en certaines grandes occasions solennelles, comme la venue d'un messager de l'empereur ou l'achèvement de grands travaux.

17. NDT : l'art de la scène appelé kagura (« danses offertes aux dieux ») se présente souvent sous la forme de danses rituelles et de musique à vocation propitiatoire en particulier lors des festivals saisonniers. À l'origine, pratiqué par les missionnaires du grand sanctuaire d'Ise, le dai-kagura devint un spectacle comportant des tours d'adresse ou d'acrobatie très répandu à l'époque d'Edo. 18. NDT : Kawakami Otojirō (1864-1911) est une célèbre figure du théâtre japonais moderne. Voir l'article de Jean-Jacques Tschudin, dans ce numéro.

19. Sont citées ici «Hachinoki, Yamanba, Yorimasa 頼政, Hyakuman 百万, Miidera 三井寺, Kashiwazaki 柏崎, Mochizuki 望月, Kasugaryūjin 春日龍神, Sanemori 実盛, ...» 
20. NDT : le mot orientarizumu est entré dans la langue japonaise sous l'influence de l'ouvrage d'Edward Saïd dont la traduction en japonais fut publiée en 1986.

21. Tetsudō jihō, $n^{\circ} 299$, huitième lettre.

22. Meiji sanjūhachinen chokurei dainihyakuyonjūsan gō 明治三十八年勅令第二百四十三号, Kankoku ni tōkanfu oyobi rijichō o oku no ken 韓国二統監府及理事庁习置クノ件.

23. NDT : sur cette recherche d'un art de la scène national qui puisse concurrencer l'opéra occidental, voir également l'article de Claude MICHEL-LESNE, dans ce numéro.

24. Le présent article constitue le premier pas d'une recherche sur l'histoire du nō et ses évolutions aussi bien au Japon que dans la Corée colonisée avec comme étapes à venir les années 1910, 1920, 1930 et 1940.

\section{RÉSUMÉS}

La crise que traversa un art de la scène tel que le nō à l'occasion des bouleversements entraînés par la restauration de Meiji peut être considérée comme un choc entre tradition et modernité. Dès lors que le nō fut reconnu comme une «tradition» par les autorités du processus de la modernisation, il put être intégré au monde moderne en acquérant le statut du « classicisme » et put ainsi se développer dans le présent et se projeter dans l'avenir. Le nō trouva ainsi une nouvelle vie en devenant une forme artistique représentative du nouvel État moderne. Ses premières représentations à l'étranger se déroulèrent en Corée avant que la péninsule ne devienne une colonie du Japon. Je me propose ici d'examiner la question du nō dans la Corée de 1905, à la veille de l'institution du gouvernement général japonais et en particulier les représentations qui furent données à l'occasion de la cérémonie d'ouverture de la ligne de chemin de fer reliant Keijō 京城 (Kyŏngsŏng en coréen, l'actuelle Seoul) à Pusan 釜山, le port coréen le plus proche du Japon.

The crisis that crossed a performing art such as nō during the upheavals caused by the Meiji Restoration can be considered as a clash between tradition and modernity. Since the nō was recognized as a "tradition" by the authorities of the process of modernization, it could be integrated into the modern world by acquiring the status of "classicism" and was thus able to develop in this project and in the future. The nō and found a new life by becoming a representative art form of the new modern state. His first performances abroad took place in Korea before the peninsula became a colony of Japan. I propose here to examine the question of nō in Korea in 1905, on the eve of the institution of the Japanese General Government and in particular the representations that were given on the occasion of the opening of the line ceremony railway linking Keijo 京城 (Kyongsong Korean, the current Seoul) 釜山 Pusan, Korean nearest port of Japan. 
INDEX

Index géographique : Corée

Thèmes : arts du spectacle

Mots-clés : colonisation, théâtre, nō, Kanze Kiyokado

Keywords : Korea, Performing Arts, Colonization, nō, Theater, Kanze Kiyokado, Meiji Period

Index chronologique : Meiji (1868-1912)

\section{AUTEURS}

JOHNG WAN SUH

Université Hallym 